\title{
Selección de ítems para el desarrollo de un instrumento de evaluación de severidad de trastornos mentales en pacientes sin psicosis o déficit cognitivo.
}

Item selection for the development of a severity assessment instrument for mental disorders in patients without psychosis or cognitive deficit.

Percy G. Damiani-Rodríguez ${ }^{1, \text { a }}$, Johann M. Vega-Dienstmaier ${ }^{1, \text { b,c }}$

\section{RESUMEN}

Objetivo: Seleccionar los ítems más relevantes para la creación de un instrumento de evaluación global de pacientes psiquiátricos ambulatorios sin psicosis ni déficit cognitivo. Material y Métodos: Se aplicaron 60 ítems, procedentes de diferentes escalas que evalúan principalmente severidad de síntomas, discapacidad y calidad de vida, a 313 pacientes con trastornos mentales de la consulta ambulatoria de psiquiatría del Hospital Cayetano Heredia (Lima, Perú). Al mismo tiempo, se evaluó la severidad de su sintomatología mental a través de la Escala de Impresión Clínica Global de Severidad (CGI-S). Se determinaron los ítems más relevantes en base a las cargas factoriales (CF) de cada ítem producto de un análisis factorial policórico y las correlaciones policóricas entre cada ítem y la CGI-S (cCGI). Resultados: Los 11 ítems más relevantes fueron: Calidad de vida global (CF=0,7958, cCGI=-0,4275), Bienestar psicológico $(\mathrm{CF}=0,7891$, cCGI $=-0,4507)$, Tristeza $(\mathrm{CF}=0,7601$, cCGI=-0,4613), Sentimientos negativos $(\mathrm{CF}=0,7595$, cCGI=-0,4269), Angustia $(\mathrm{CF}=0,7501$, cCGI $=-0,4824)$, Libertad y seguridad $(\mathrm{CF}=0,7402$, cCGI $=$ -0,4011), Suicidalidad ( $\mathrm{CF}=0,7147$, cCGI=-0,4766), Sentido de vida $(\mathrm{CF}=0,6998$, cCGI=-0,4161), Crecimiento personal $(\mathrm{CF}=0,6987, \mathrm{cCGI}=-0,4198)$, Espiritualidad, religión y creencias personales $(\mathrm{CF}=0,6936, \mathrm{cCGI}=-0,3925)$ y Plenitud personal ( $\mathrm{CF}=0,6869$, cCGI=-0,4073). Conclusiones: Los 11 ítems seleccionados podrían constituir un instrumento corto de auto-reporte para la evaluación de enfermedad mental.

PALABRAS CLAVE: Calidad de vida, bienestar, salud mental, discapacidad, psicometría.

\section{SUMMARY}

Objective: To select the most relevant items for the creation of a global evaluation instrument for psychiatric outpatients without psychosis or cognitive impairment. Methods: 313 psychiatric outpatients from Hospital Cayetano Heredia (Lima, Peru) completed 60 items taken from a variety of scales focused on measuring severity of symptoms, disability, and quality of life. At the same time, the severity of their mental symptoms was assessed through the Clinical Global Impressions Scale (CGI-S). The most relevant items were chosen based on each item's factorial loads (FL) resulting from a polychoric factorial analysis and the polychoric correlations between each item

\footnotetext{
Facultad de Medicina Alberto Hurtado, Universidad Peruana Cayetano Heredia. Lima, Perú.

Bachiller en medicina.

Médico-Psiquiatra.

c Maestro en epidemiología clínica.
} 
and the CGI-S (CGIc). Results: The 11 most relevant items were: Global perception of quality of life ( $\mathrm{FL}=0.7958$, $\mathrm{CGIc}=-0.4275)$, Psychological/Emotional wellbeing ( $\mathrm{FL}=0.7891$, CGIc $=-0.4057)$, Sadness $(\mathrm{FL}=0.7601$, CGIc $=$ $-0.4613)$, Negative feelings $(\mathrm{FL}=0.7595$, CGIc $=-0.4269)$, Angst $(\mathrm{FL}=0.7501$, CGIc $=-0.4824)$, Freedom and physical safety $(\mathrm{FL}=0.7402$, $\mathrm{CGI}=-0.4011)$, Suicidality $(\mathrm{FL}=0.7147$, CGIc=-0.4766), Life meaning $(\mathrm{FL}=0.6998$, $\mathrm{CGIc}=$ $-0.4161)$, Personal growth ( $\mathrm{FL}=0.6987$, CGIc $=-0.4198)$, Spirituality and personal beliefs $(\mathrm{FL}=0.6936$, CGIc $=$ -0.3925), and Personal fulfillment ( $\mathrm{FL}=0.6869, \mathrm{CGIc}=-0.4073)$. Conclusion: The 11 selected items could constitute a short self-reporting instrument for the evaluation of mental illness.

KEYWORDS: Quality of life, wellbeing, mental health, disability, psychometrics.

\section{INTRODUCCIÓN}

Las enfermedades neuro-psiquiátricas constituyen la primera causa de carga de enfermedad en el Perú, alcanzando el 17,4 \% del total de años de vida saludable perdidos, principalmente debido al componente de discapacidad (1). La prevalencia anual de uno o más trastornos de salud mental en el 2005 en Lima y otras cuatro ciudades de Perú fue de 13,5\% según el "Estudio Mundial de la Salud Mental, 2005", siendo catalogados como enfermedad mental leve el $41,2 \%$, como moderados el $41,6 \%$ y como severos el $17,2 \%$ de los casos, de acuerdo al criterio establecido por la Composite International Diagnostic Interview (2). Por otro lado, en el 2012, la prevalencia de vida para cualquier trastorno mental fue de $26,1 \%$ y la prevalencia a 12 meses de $11,8 \%$ en Lima Metropolitana y Callao (3). Es por esta situación que cobra aún más importancia el diagnóstico y seguimiento de los trastornos mentales.

En la actualidad la valoración del estado de la enfermedad psiquiátrica, se da principalmente a través de la evaluación y apreciación clínica del psiquiatra tratante, la cual puede ser guiada por entrevistas estructuradas o escalas como la Clinical Global Impression Scale (CGI) (4). Para la evaluación consistente de la severidad de los trastornos psiquiátricos es necesario contar primero con una definición clara de lo que califica como una enfermedad mental severa. Se han propuesto distintos criterios y definiciones, sin embargo no existe un consenso generalizado con respecto a una definición operacional (5); algunas de las dimensiones descritas son, por ejemplo, la severidad de síntomas, la existencia de comorbilidades (6) y la disfunción causada por la enfermedad $(7,8)$.

El factor común entre la mayoría de las definiciones propuestas, como las planteadas por el Substance Abuse and Mental Health Services Association's National Registry of Evidence-Based Programs and Practices en Estados Unidos (9), la Organización Mundial de la Salud (10) y el National Institute of Mental Health de Estados Unidos (11), es que toman en cuenta que la enfermedad interfiere de forma significativa con el desarrollo de las actividades mayores de la vida (12).

Actualmente se plantea la noción de que los trastornos psiquiátricos ocurren a lo largo de un espectro, en vez de encontrarse cada uno encasillado como una entidad independiente (13). La presencia de discapacidad, definida como la dificultad para funcionar a nivel de cuerpo, persona o sociedad, en uno o más dominios de la vida, como es experimentada por un individuo con una condición de salud en interacción con factores contextuales, es un factor transversal, en el sentido de que no discrimina etiologías, permitiendo que distintas enfermedades sean evaluadas bajo la misma luz (14). Describiendo el estado de salud en base a la percepción del sujeto, de su posición en la vida en el contexto de su cultura y sistemas de valores en relación a sus metas y preocupaciones, y la interacción entre su condición de salud y factores personales y ambientales, se puede obtener una visión más amplia de la condición del paciente, enfocándolo desde una perspectiva bio-psico-social $(15,16)$.

A la fecha se han validado una serie de instrumentos los cuales permiten clasificar el estado del paciente con respecto a las dimensiones mencionadas anteriormente como el World Health Organization Disability Assessment Schedule (WHODAS) (17), el World Health Organization Quality of Life Instrument (WHOQOL) (18), el Índice de Calidad de Vida (ICV) $(19,20)$, el Pemberton Happiness Index (PHI) (21) y las medidas de síntomas transversales del DSM-5 (22), y se ha establecido una clara correlación entre el deterioro de estas dimensiones y el declive de la enfermedad mental (8).

Además existen instrumentos validados en el Perú tales como la Escala de Ansiedad de Lima (EAL) (23) y la Escala de Psicopatología Depresiva (EPD) (24), 
Selección de ítems para el desarrollo de un instrumento de evaluación de severidad de trastornos mentales.

las cuales evalúan respectivamente trastornos ansiosos y depresivos, considerados como los más prevalentes entre las enfermedades mentales. Asimismo, la depresión es uno de los mayores contribuyentes a la carga de discapacidad por enfermedades en general y ocurre frecuentemente de forma concomitante a otras enfermedades tanto físicas como mentales (25).

La ausencia de una escala estandarizada que permita la valoración global del estado actual de un paciente en seguimiento es lo que motiva el presente estudio que busca seleccionar ítems tomados de las escalas mencionadas para desarrollar un cuestionario corto y auto-aplicable que tenga como pilares principales la evaluación de la severidad de síntomas, la disfunción causada por la enfermedad y el impacto que tiene sobre la calidad de vida.

El objetivo del estudio fue identificar los ítems más relevantes de las escalas seleccionadas, para desarrollar un instrumento de evaluación global del estado del trastorno psiquiátrico basado en la calidad de vida, severidad de los síntomas y disfunción ocasionada por ellos, en pacientes ambulatorios con trastornos mentales sin psicosis o déficit cognitivo.

\section{MATERIAL Y MÉTODOS}

El estudio fue de tipo observacional y transversal. Se utilizó una muestra de 313 pacientes que acudían al consultorio de psiquiatría del Hospital Cayetano Heredia $(\mathrm{HCH})$, de ambos sexos, de 18 años a 65 años de edad, con grado de instrucción de al menos primaria completa y con diagnóstico de algún trastorno mental pero sin trastornos neurocognitivos (demencia, delirio), trastornos psicóticos o retraso mental. Se considera este número un buen tamaño muestral para el análisis factorial de acuerdo a Comrey y Lee (26).

\section{Selección de 60 ítems para evaluar disfuncionalidad, calidad de vida y severidad de la sintomatología}

Los ítems seleccionados y aplicados a los pacientes fueron tomados de 7 escalas las cuales han sido validadas en español y son utilizadas para medir distintos aspectos de enfermedad y su impacto en la vida del paciente. De cada escala se tomó el mayor número de ítems que fueran considerados relevantes y los cuales no fueran redundantes con respecto a los seleccionados previamente. Los 60 ítems son presentados en la tabla 1, la cual muestra la definición de las variables.
Las 7 escalas utilizadas fueron:

a) WHODAS 2.0: instrumento de 36 ítems, autoadministrable para evaluación de salud y discapacidad. Se seleccionaron los 12 ítems que conforman la versión abreviada de la escala (17).

b) WHOQOL-BREF: instrumento de 26 ítems utilizado para evaluar calidad de vida en base a salud física y psicológica, y relaciones sociales y con el ambiente. Se excluyó el ítem 10 debido a que hace referencia al dominio de salud física. Los ítems 18, 19 y 20 no fueron incluidos debido a que se estima que la información que podrían brindar es abarcada por el ICV (18).

c) ICV: escala de 10 ítems valorados del 1 al 10 para evaluación de calidad de vida. Se decidió emplear los 10 ítems que componen la escala $(19,20)$.

d) Medidas de Sintomas Transversales del DSM-5: consta de 23 preguntas para evaluación de 13 distintos dominios psiquiátricos. Se tomaron tres preguntas: 3, 11 y 14; que evalúan sintomatología relacionada con irritabilidad, agresividad y sueño (22).

e) Escala de Psicopatología Depresiva: instrumento de 20 ítems para evaluación de depresión. Fueron seleccionados 4 ítems considerados los más representativos para la evaluación de depresión mayor (24).

f) Escala de Ansiedad de Lima: escala de 130 ítems para evaluación de sintomatología de trastornos de ansiedad. Se seleccionaron los 5 ítems más representativos de ansiedad psíquica, fobia social, ansiedad física y agorafobia (23).

g) El Pemberton Happines Index (PHI): escala de 21 ítems la cual evalúa el bienestar utilizando los dominios de bienestar recordado y bienestar experimentado. Se utilizaron 8 preguntas del cuestionario: 4 de bienestar recordado y 4 de bienestar experimentado (21).

Por otro lado, el psiquiatra tratante del paciente aplicó la Escala de Impresión Clínica Global de Severidad (CGI-S). Esta escala consta de 7 categorías de severidad que van desde "normal" hasta "entre los pacientes más enfermos"; fue desarrollada para proveer una evaluación breve e independiente del estado de funcionamiento global del paciente desde la 
perspectiva del clínico, utilizando toda la información disponible, incluyendolahistoria clínica, circunstancias psicosociales, síntomas, comportamiento e impacto de los síntomas sobre el funcionamiento (4).

\section{Análisis de datos y selección de items}

Se elaboró una matriz de correlaciones policóricas entre los 60 ítems aplicados, a partir de la cual se realizó un análisis factorial exploratorio utilizando la rotación varimax; asimismo, se elaboró el gráfico de sedimentación de Cattel correspondiente para evaluar el número de factores relevantes (27); adicionalmente, se calcularon los coeficientes de correlación policóricos entre cada ítem del instrumento y la CGI-S.

Se ordenaron los ítems de acuerdo a sus cargas factoriales respecto al factor único identificado en el análisis factorial y según la fuerza de correlación con la CGI-S; y considerando estas dos formas de ordenamiento, se planeó escoger los 10 mejores ítems. Se formaron dos conjuntos: uno, con aquellos ítems con mayor carga factorial (CF); y otro, con los que presentaron una mayor correlación con la CGI-S. Se fue aumentando el número de ítems en cada conjunto hasta alcanzar al menos 10 ítems comunes a ambos conjuntos. Los análisis se llevaron a cabo con STATA v. 14.

El protocolo de investigación fue aprobado por el Comité de Ética de la Universidad Peruana Cayetano Heredia y el Comité de Ética del HCH. Los pacientes firmaron un consentimiento informado aceptando participar en el estudio.

\section{RESULTADOS}

Se evaluó una muestra de 313 pacientes cuyas características se muestran en la tabla 2; la mayoría fueron mujeres, la edad promedio fue 42,31 años con una desviación estándar de 14,12; y los percentiles 25,50 y 75 fueron: 29, 44 y 54, respectivamente. La mayor parte de los pacientes contaba con educación secundaria completa. Las ocupaciones más comunes fueron: ama de casa, estudiante, sin ocupación, comerciante e independiente. Con respecto a la severidad de la enfermedad psiquiátrica diagnosticada en cada paciente, cuantificada por medio de la CGI-S, se encontró un valor promedio de 3,54; siendo el grupo más grande el calificado con "4" (moderadamente enfermo), seguido por " 3 " (levemente enfermo) y " 5 " (marcadamente enfermo).
Se diagnosticaron 49 distintas patologías, de las cuales las más frecuentes fueron episodio depresivo (F32), trastorno de ansiedad sin especificación (F41.9), trastorno mixto ansioso-depresivo (F41.2), trastorno de ansiedad generalizada (F41.1), trastorno de pánico (F41.0) y trastorno de inestabilidad emocional de la personalidad (F60.3), patologías que representaron el $88,58 \%$ de los diagnósticos. En la tabla 3 se muestran los diagnósticos encontrados.

En las tablas 4 y 5 se observa la evaluación de la relación entre el sexo del paciente y el puntaje asignado en cada pregunta, tanto en ítems de más de 4 opciones como ítems de menos de 5 opciones, respectivamente. Se encontró que existen diferencias significativas en relación al sexo en los ítems: dificultad para caminar, dificultad para concentrarse, sentimiento de seguridad, ambiente físico, calidad de la salud física, disponibilidad de tiempo para actividades de ocio, disponibilidad de servicios de salud, bienestar físico, sentimientos de enfado, problemas de sueño, cansancio, falta de aire y miedo a caminar por la calle. Además, la tabla 4 muestra los puntajes promedio por ítem y la desviación estándar en los ítems con más de 4 opciones; y la tabla 5, el porcentaje por respuesta en cada ítem con menos de 5 alternativas.

En el gráfico de sedimentación se observa el tamaño relativo de los valores propios por factor, encontrándose una caída brusca después del Factor 1, luego de la cual no hay disminuciones importantes de los valores propios; esto sugiere que hay que retener solo 1 factor en el análisis (gráfico 1)(26).

Por medio del análisis factorial se encontraron los ítems con mayor correlación con el factor único al que vamos a denominar "salud". Los tres ítems con mayor carga factorial (CF) fueron: el ítem 40, calidad de vida global; el ítem 32, bienestar psicológico; y el ítem 46 , tristeza. Los ítems con mayor correlación con el puntaje de la CGI-S asignado por el médico evaluador fueron: el ítem 50, disnea; el ítem 52, miedo a caminar por la calle; y el ítem 49, angustia. En la tabla 6 se presentan los valores de las cargas factoriales y de la correlación con el CGI-S por cada ítem.

Se formaron dos conjuntos tomando en uno los ítems con las mayores CF y en el segundo los ítems con mayores valores de correlación con la CGI-S. Se estableció como punto de corte inicial para cada grupo 15 ítems, valor con el que se encontró un total de 9 ítems presentes en ambos conjuntos. Al considerar los 16 ítems más relevantes de cada conjunto se obtuvo 
Selección de ítems para el desarrollo de un instrumento de evaluación de severidad de trastornos mentales.

el mismo resultado, habiendo nuevamente solo 9 ítems presentes. Finalmente, al tomar los 17 ítems más relevantes de cada conjunto se encontró un total de 11 ítems en común; los ítems: 40 (Calidad de vida global), 32 (Bienestar psicológico), 46 (Tristeza), 30 (Sentimientos negativos), 49 (Angustia), 17 (Libertad y seguridad), 42 (Suicidalidad), 53 (Sentido de vida), 54 (Crecimiento personal), 15 (Espiritualidad, religión y creencias personales) y 38 (Plenitud personal). En la tabla 7 se presentan los 17 ítems más relacionados de cada conjunto resaltando los 11 encontrados en los dos conjuntos.

Tabla 1. Definición de variables.

\begin{tabular}{|c|c|c|c|}
\hline NOMBRE & DEFINICIÓN & TIPO & VALORES \\
\hline Estar de pie $(01)$ & Ítem S1-WHODAS 2.0 & Ordinal & $1-5$ \\
\hline Quehaceres (02) & Ítem S2-WHODAS 2.0 & Ordinal & $1-5$ \\
\hline Aprender una nueva tarea $(03)$ & Ítem S3-WHODAS 2.0 & Ordinal & $1-5$ \\
\hline Problemas en comunidades (04) & Ítem S4-WHODAS 2.0 & Ordinal & $1-5$ \\
\hline Haber sido afectado emocionalmente (05) & Ítem S5-WHODAS 2.0 & Ordinal & $1-5$ \\
\hline Concentrarse (06) & Ítem S6-WHODAS 2.0 & Ordinal & $1-5$ \\
\hline Andar (07) & Ítem S7-WHODAS 2.0 & Ordinal & $1-5$ \\
\hline Lavarse (08) & Ítem S8-WHODAS 2.0 & Ordinal & $1-5$ \\
\hline Vestirse (09) & Ítem S9-WHODAS 2.0 & Ordinal & $1-5$ \\
\hline Mantener amistad (11) & Ítem S11-WHODAS 2.0 & Ordinal & $1-5$ \\
\hline Trabajo diario/Actividades escolares (12) & Ítem S12-WHODAS 2.0 & Ordinal & $1-5$ \\
\hline Dependencia de medicinas (13) & Ítem 4- WHOQOL-BREF & Ordinal & $1-5$ \\
\hline Sentimientos positivos (14) & Ítem 5-WHOQOL- BREF & Ordinal & $1-5$ \\
\hline Espiritualidad, religión, creencias personales (15) & Ítem 6-WHOQOL-BREF & Ordinal & $1-5$ \\
\hline Pensamiento, aprendizaje, memoria, concentración (16) & Ítem 7-WHOQOL- BREF & Ordinal & $1-5$ \\
\hline Libertad y seguridad (17) & Ítem 8-WHOQOL- BREF & Ordinal & $1-5$ \\
\hline Ambiente físico (18) & Ítem 9-WHOQOL- BREF & Ordinal & $1-5$ \\
\hline Imagen corporal (19) & Ítem 11-WHOQOL- BREF & Ordinal & $1-5$ \\
\hline Recursos económicos (20) & Ítem 12-WHOQOL- BREF & Ordinal & $1-5$ \\
\hline Oportunidad de información (21) & Ítem 13-WHOQOL- BREF & Ordinal & $1-5$ \\
\hline Ocio y descanso (22) & Ítem 14-WHOQOL- BREF & Ordinal & $1-5$ \\
\hline Movilidad (23) & Ítem 15-WHOQOL- BREF & Ordinal & $1-5$ \\
\hline Sueño y descanso (24) & Ítem 16-WHOQOL- BREF & Ordinal & $1-5$ \\
\hline Relaciones personales (25) & Ítem 17-WHOQOL- BREF & Ordinal & $1-5$ \\
\hline Actividad sexual (26) & Ítem 21-WHOQOL- BREF & Ordinal & $1-5$ \\
\hline Hogar (27) & Ítem 23-WHOQOL- BREF & Ordinal & $1-5$ \\
\hline Atención sanitaria / social (28) & Ítem 24-WHOQOL- BREF & Ordinal & $1-5$ \\
\hline Transporte (29) & Ítem 25-WHOQOL- BREF & Ordinal & $1-5$ \\
\hline Sentimientos negativos (30) & Ítem 26-WHOQOL- BREF & Ordinal & $1-5$ \\
\hline
\end{tabular}


Tabla 1. Definición de variables. (continuación)

\begin{tabular}{|c|c|c|c|}
\hline NOMBRE & DEFINICIÓN & TIPO & VALORES \\
\hline Bienestar físico (31) & Ítem 1-ICV & Ordinal & $1-10$ \\
\hline Bienestar psicológico (32) & Ítem 2-ICV & Ordinal & $1-10$ \\
\hline Autocuidado (33) & Ítem 3-ICV & Ordinal & $1-10$ \\
\hline Funcionamiento ocupacional (34) & Ítem 4-ICV & Ordinal & $1-10$ \\
\hline Funcionamiento interpersonal (35) & Ítem 5-ICV & Ordinal & $1-10$ \\
\hline Apoyo social-emocional (36) & Ítem 6-ICV & Ordinal & $1-10$ \\
\hline Apoyo comunitario y de servicios (37) & Ítem 7-ICV & Ordinal & $1-10$ \\
\hline Plenitud personal (38) & Ítem 8-ICV & Ordinal & $1-10$ \\
\hline Satisfacción espiritual (39) & Ítem 9-ICV & Ordinal & $1-10$ \\
\hline Calidad de vida global (40) & Ítem 10-ICV & Ordinal & $1-10$ \\
\hline Enfado (41) & $\begin{array}{l}\text { Ítem 3-DSM-5 Medidas de síntomas } \\
\text { transversales }\end{array}$ & Ordinal & $1-5$ \\
\hline Suicidalidad (42) & $\begin{array}{l}\text { Ítem 11-DSM-5 Medidas de } \\
\text { síntomas transversales }\end{array}$ & Ordinal & $1-5$ \\
\hline Sueño (43) & $\begin{array}{l}\text { Ítem 14-DSM-5 Medidas de } \\
\text { síntomas transversales }\end{array}$ & Ordinal & $1-5$ \\
\hline Cansado (44) & Ítem 5-EPD & Ordinal & $1-4$ \\
\hline Anhedonia (45) & Ítem 7-EPD & Ordinal & $1-4$ \\
\hline Tristeza (46) & Ítem 15-EPD & Ordinal & $1-4$ \\
\hline Reactividad (47) & Ítem 17-EPD & Ordinal & $1-4$ \\
\hline Nerviosismo (48) & Ítem 14-EAL & Dicotómica & Sí o No \\
\hline Angustia (49) & Ítem 19-EAL & Dicotómica & Sí o No \\
\hline Disnea $(50)$ & Ítem 21-EAL & Dicotómica & Sí o No \\
\hline Grupo (51) & Ítem 28-EAL & Dicotómica & Sí o No \\
\hline Calle (52) & Ítem 17-EAL & Dicotómica & Sí o No \\
\hline Sentido de vida (53) & Ítem R3-PHI & Ordinal & $1-10$ \\
\hline Crecimiento personal (54) & Ítem R5-PHI & Ordinal & $1-10$ \\
\hline Control percibido (55) & Ítem R7-PHI & Ordinal & $1-10$ \\
\hline Autonomía (56) & Ítem R8-PHI & Ordinal & $1-10$ \\
\hline Experiencia positiva - Orgullo (57) & Ítem E1-PHI & Dicotómica & Sí o No \\
\hline Experiencia negativa- Abrumado (58) & Ítem E2-PHI & Dicotómica & Sí o No \\
\hline Experiencia positiva - Aprender (59) & Ítem E7-PHI & Dicotómica & Sí o No \\
\hline Experiencia negativa- Menospreciado (60) & Ítem E10-PHI & Dicotómica & Sí o No \\
\hline
\end{tabular}


Selección de ítems para el desarrollo de un instrumento de evaluación de severidad de trastornos mentales.

Tabla 2. Características de la muestra.

\begin{tabular}{lcc}
\hline VARIABLE & n & \% \\
\hline Sexo & & \\
Femenino & 222 & 70,93 \\
Masculino & 91 & 29,07 \\
Edad & & \\
$18-20$ & 17 & 5,43 \\
$21-30$ & 67 & 21,41 \\
$31-40$ & 48 & 15,34 \\
$41-50$ & 72 & 23,00 \\
$51-60$ & 73 & 23,32 \\
$61-65$ & 36 & 11,50
\end{tabular}

\section{Grado de instrucción}

Superior completa

Superior incompleta

Técnica completa

Técnica incompleta

Secundaria completa

Secundaria incompleta

Primaria completa

\section{Ocupación}

Ama de casa

Estudiante

Comerciante

Independiente

Otros

Sin ocupación

\section{Severidad de enfermedad}

Normal, no enfermo (1)

Dudosamente enfermo (2)

Levemente enfermo (3)

Moderadamente enfermo (4)

Marcadamente enfermo (5)

Gravemente enfermo (6)

Entre los pacientes más extremadamente enfermos (7)

$$
\begin{array}{cc}
71 & 22,68 \\
13 & 4,15 \\
33 & 10,54 \\
3 & 0,96 \\
157 & 50,16 \\
15 & 4,79 \\
21 & 6,71
\end{array}
$$

$109 \quad 34,94$

$46 \quad 14,74$

$13 \quad 4,17$

$11 \quad 3,53$

$100 \quad 32,04$

$33 \quad 10,58$

$\begin{array}{cc}16 & 5,35 \\ 21 & 7,02 \\ 86 & 28,76 \\ 140 & 46,82 \\ 34 & 11,37 \\ 2 & 0,67 \\ 0 & 0\end{array}$


Tabla 3. Diagnósticos psiquiátricos de los participantes.

\section{DIAGNÓSTICO}

Episodios depresivos

Episodio depresivo (F32)

Episodio depresivo leve (F32.0)

Episodio depresivo moderado (F32.1)

Episodio depresivo severo, sin síntomas psicóticos (F32.2)

Episodio depresivo severo, con síntomas psicóticos (F32.3)

Otros trastornos de ansiedad

Trastorno de pánico (F41.0)

Trastorno de ansiedad generalizada (F41.1)

Trastorno mixto ansioso-depresivo (F41.2)

Trastorno de ansiedad sin especificación (F41.9)

Trastornos de ansiedad fóbica

Agorafobia (F40.0)

Fobias sociales (F40.1)

Fobias especificas (F40.2)

Trastornos específicos de la personalidad

Trastorno de inestabilidad emocional de la personalidad (F60.3)

Otros trastornos específicos de la personalidad

Trastorno bipolar

Trastorno bipolar (F31)

Trastornos mentales y del comportamiento debido al consumo de sustancias psicotropas

Trastornos mentales y del comportamiento debidos al consumo de cannabinoides, consumo perjudicial (F12.1)

Trastornos mentales y del comportamiento debidos al consumo de múltiples drogas o de otras sustancias psicotropas, síndrome de dependencia (F19.2)

Otros trastornos mentales y del comportamiento debido al consumo de sustancias psicotropas

Trastorno obsesivo-compulsivo

Trastorno obsesivo-compulsivo (F42)

Trastorno obsesivo-compulsivo con predominio de pensamientos o rumiaciones obsesivas (F42.0)

Otros trastornos de partes blandas y los no especificados, no clasificados bajo otro concepto

Fibromialgia (M79.7)

Trastornos somatomorfos

Trastorno de somatización (F45.0)

Trastorno hipocondriaco (F45.2)

Trastorno depresivo recurrente

Trastorno depresivo recurrente (F33)

Otros n \% DIAGNOSTICADO

41,28

$\begin{array}{cc}63 & 20 \\ 1 & 0,32 \\ 13 & 4,13 \\ 48 & 15,24 \\ 5 & 1,59 \\ & \mathbf{4 1 , 5 9} \\ 28 & 8,89 \\ 31 & 9,84 \\ 33 & 10,48 \\ 39 & 12,38\end{array}$

7,61

1,90

4,44

1,27

6,99

18

5,71

4

1,28

4,76

15

4,76

4,44

0,95

0,95

2,56

2,86

2,54

0,32

2,86

2,86

$\mathbf{2 , 8 5}$

0,63

2,22

1,90

6

1,90

17 
Selección de ítems para el desarrollo de un instrumento de evaluación de severidad de trastornos mentales.

Tabla 4. Descripción de ítems con más de 4 alternativas.

\begin{tabular}{|c|c|c|c|c|c|c|c|c|}
\hline \multirow[t]{2}{*}{ ÍTEM } & \multicolumn{3}{|c|}{ MEDIA } & \multirow{2}{*}{$\begin{array}{c}\mathbf{P}^{*} \\
(\mathrm{MWU})\end{array}$} & \multirow[t]{2}{*}{ MEDIANA } & \multirow[t]{2}{*}{ DE } & \multirow[t]{2}{*}{$\mathbf{P} 25$} & \multirow[t]{2}{*}{ P75 } \\
\hline & TOTAL & $\mathbf{H}^{* *}$ & $\mathbf{M} * * *$ & & & & & \\
\hline Estar de pie (01) & 1,26 & 1,09 & 1,33 & 0,1561 & 1 & 1,10 & 0 & 2 \\
\hline Quehaceres (02) & 1,42 & 1,34 & 1,45 & 0,3119 & 1 & 1,11 & 0 & 2 \\
\hline Aprender una nueva tarea $(03)$ & 1,12 & 1,02 & 1,16 & 0,4739 & 1 & 1,16 & 0 & 2 \\
\hline Problemas en comunidades (04) & 1,27 & 1,35 & 1,25 & 0,4268 & 1 & 1,21 & 0 & 2 \\
\hline Haber sido afectado emocionalmente (05) & 2,13 & 2,01 & 2,19 & 0,2169 & 2 & 1,02 & 1 & 3 \\
\hline Concentrarse (06) & 1,35 & 1,17 & 1,43 & 0,0693 & 1 & 1,09 & 0 & 2 \\
\hline Andar (07) & 1,30 & 0,86 & 1,48 & $<0,001$ & 1 & 1,22 & 0 & 2 \\
\hline Lavarse (08) & 0,68 & 0,60 & 0,72 & 0,3694 & 0 & 0,93 & 0 & 1 \\
\hline Vestirse (09) & 0,62 & 0,48 & 0,69 & 0,1316 & 0 & 0,88 & 0 & 1 \\
\hline Relacionarse con desconocidos (10) & 0,99 & 1,00 & 1,00 & 0,8126 & 1 & 1,10 & 0 & 2 \\
\hline Mantener amistad (11) & 0,95 & 0,98 & 0,94 & 0,9658 & 1 & 1,09 & 0 & 2 \\
\hline Trabajo diario / Actividades escolares (12) & 1,24 & 1,28 & 1,24 & 0,9848 & 1 & 1,13 & 0 & 2 \\
\hline Dependencia de medicinas (13) & 2,96 & 3,12 & 2,91 & 0,0875 & 3 & 0,94 & 2 & 4 \\
\hline Sentimientos positivos (14) & 2,59 & 2,71 & 2,55 & 0,0868 & 3 & 0,81 & 2 & 3 \\
\hline Espiritualidad, religión, creencias personales (15) & 2,98 & 3,15 & 2,93 & 0,0863 & 3 & 0,91 & 2 & 4 \\
\hline Pensamiento, aprendizaje, memoria, concentración (16) & 2,58 & 2,72 & 2,51 & 0,0136 & 3 & 0,74 & 2 & 3 \\
\hline Libertad y seguridad (17) & 2,70 & 2,90 & 2,62 & 0,0032 & 3 & 0,84 & 2 & 3 \\
\hline Ambiente físico (18) & 2,79 & 2,97 & 2,72 & 0,0228 & 3 & 0,78 & 2 & 3 \\
\hline Imagen corporal (19) & 3,00 & 3,27 & 2,89 & 0,0083 & 3 & 2,02 & 2 & 3 \\
\hline Recursos económicos (20) & 2,33 & 2,42 & 2,30 & 0,1739 & 2 & 0,75 & 2 & 3 \\
\hline Oportunidad de información (21) & 2,74 & 2,80 & 2,71 & 0,5667 & 3 & 1,04 & 2 & 3 \\
\hline Ocio y descanso (22) & 2,62 & 2,85 & 2,52 & 0,0017 & 3 & 0,87 & 2 & 3 \\
\hline Movilidad (23) & 3,33 & 3,49 & 3,27 & 0,0719 & 3 & 1,07 & 3 & 4 \\
\hline Sueño y descanso (24) & 2,49 & 2,73 & 2,39 & 0,006 & 2 & 0,94 & 2 & 3 \\
\hline Relaciones personales (25) & 2,64 & 2,73 & 2,60 & 0,1645 & 3 & 0,83 & 2 & 3 \\
\hline Actividad sexual (26) & 2,53 & 2,59 & 2,50 & 0,5897 & 3 & 0,92 & 2 & 3 \\
\hline Hogar (27) & 2,82 & 2,99 & 2,75 & 0,0503 & 3 & 0,99 & 2 & 3 \\
\hline Atención sanitaria / social (28) & 2,87 & 3,03 & 2,79 & 0,0192 & 3 & 1,00 & 2 & 3 \\
\hline Transporte (29) & 2,35 & 2,50 & 2,28 & 0,0622 & 2 & 1,46 & 2 & 3 \\
\hline Sentimientos negativos (30) & 2,60 & 2,77 & 2,52 & 0,0914 & 3 & 0,99 & 2 & 3 \\
\hline Bienestar físico (31) & 5,06 & 5,65 & 4,81 & 0,004 & 5 & 2,34 & 3 & 7 \\
\hline Bienestar psicológico (32) & 5,44 & 5,74 & 5,31 & 0,1597 & 5 & 2,52 & 3 & 7,25 \\
\hline Autocuidado (33) & 6,78 & 7,20 & 6,60 & 0,0634 & 7 & 2,44 & 5 & 9 \\
\hline Funcionamiento ocupacional (34) & 6,82 & 6,97 & 6,79 & 0,4887 & 7 & 2,44 & 5 & 9 \\
\hline Funcionamiento interpersonal (35) & 6,95 & 7,02 & 6,92 & 0,9441 & 7 & 2,41 & 5 & 9 \\
\hline Apoyo social-emocional (36) & 6,79 & 7,03 & 6,69 & 0,5352 & 7,5 & 2,62 & 5 & 9 \\
\hline
\end{tabular}


Damiani-Rodríguez P, Vega-Dienstmaier J.

Tabla 4. Descripción de ítems con más de 4 alternativas. (continuación)

\begin{tabular}{|c|c|c|c|c|c|c|c|c|}
\hline \multirow[t]{2}{*}{ ÍTEM } & \multicolumn{3}{|c|}{ MEDIA } & \multirow[t]{2}{*}{$\begin{array}{c}\mathbf{P}^{*} \\
(\mathbf{M W U})\end{array}$} & \multirow[t]{2}{*}{ MEDIANA } & \multirow[t]{2}{*}{ DE } & \multirow[t]{2}{*}{$\mathbf{P} 25$} & \multirow[t]{2}{*}{ P75 } \\
\hline & TOTAL & $\mathbf{H}^{* *}$ & $\mathbf{M}^{* * *}$ & & & & & \\
\hline Apoyo comunitario y de servicios (37) & 4,94 & 5,29 & 4,80 & 0,1346 & 5 & 2,72 & 3 & 7 \\
\hline Plenitud personal (38) & 5,60 & 5,93 & 5,46 & 0,1736 & 6 & 2,71 & 3 & 8 \\
\hline Satisfacción espiritual (39) & 6,51 & 6,38 & 6,57 & 0,5394 & 7 & 2,50 & 5 & 8 \\
\hline Calidad de vida global (40) & 6,17 & 6,63 & 5,99 & 0,0661 & 6 & 2,60 & 4 & 8 \\
\hline Enfado (41) & 1,91 & 1,69 & 2,00 & 0,0329 & 2 & 1,17 & 1 & 3 \\
\hline Suicidalidad (42) & 0,60 & 0,59 & 0,61 & 0,8993 & 0 & 2,52 & 0 & 1 \\
\hline Sueño (43) & 1,62 & 1,30 & 1,76 & 0,0024 & 1 & 2,44 & 1 & 2 \\
\hline Sentido de vida (53) & 7,56 & 7,66 & 7,50 & 0,7178 & 8 & 2,66 & 5 & 10 \\
\hline Crecimiento personal (54) & 7,35 & 7,76 & 7,17 & 0,0937 & 8 & 2,62 & 5 & 10 \\
\hline Control percibido (55) & 6,91 & 7,40 & 6,73 & 0,0963 & 8 & 2,73 & 5 & 9 \\
\hline Autonomía (56) & 7,40 & 7,57 & 7,30 & 0,5382 & 8 & 2,56 & 5,75 & 10 \\
\hline
\end{tabular}

*Prueba U de Mann-Whitney

**Hombres

***Mujeres

Tabla 5. Descripción de ítems con menos de 5 alternativas.

\begin{tabular}{|c|c|c|c|c|}
\hline ÍTEM & $\begin{array}{c}\% \text { POR } \\
\text { ALTERNATIVA }\end{array}$ & $\begin{array}{c}\text { \% POR ALTERNATIVA } \\
\text { H** }^{* *}\end{array}$ & $\begin{array}{c}\text { \% POR ALTERNATIVA } \\
\mathrm{M}^{* * *}\end{array}$ & $\mathbf{P} *$ \\
\hline Cansado (44) & & & & $<0,001$ \\
\hline 0. Casi nunca & 10,00 & 6,00 & 4,00 & \\
\hline 1. A veces & 46,67 & 15,67 & 31,00 & \\
\hline 2. Frecuentemente & 24,67 & 5,00 & 19,67 & \\
\hline 3. Casi todo el tiempo & 18,67 & 2,67 & 16,00 & \\
\hline Anhedonia (45) & & & & 0,6245 \\
\hline 0. Sí, generalmente & 26,33 & 7,67 & 18,67 & \\
\hline 1. Algunas veces & 52,33 & 14,33 & 38,00 & \\
\hline 2. Rara vez & 14,33 & 5,67 & 8,67 & \\
\hline 3. No, en ningún momento & 7,00 & 1,67 & 5,33 & \\
\hline Triste (46) & & & & 0,0052 \\
\hline 0. Casi nunca & 19,33 & 6,67 & 12,67 & \\
\hline 1. A veces & 48,33 & 17,00 & 31,33 & \\
\hline 2. Frecuentemente & 21,00 & 4,33 & 16,67 & \\
\hline 3. Casi todo el tiempo & 11,33 & 1,33 & 10,00 & \\
\hline Reactividad (47) & & & & 0,6307 \\
\hline $0 . \mathrm{Si}$, generalmente & 66,67 & 20,33 & 46,33 & \\
\hline 1. Alguna veces & 24,00 & 6,00 & 18,00 & \\
\hline 2. Rara vez & 8,67 & 2,67 & 6,00 & \\
\hline 3. No, en ningún momento & 0,67 & 0,33 & 0,33 & \\
\hline
\end{tabular}

Continúa... 
Selección de ítems para el desarrollo de un instrumento de evaluación de severidad de trastornos mentales.

Tabla 5. Descripción de ítems con menos de 5 alternativas. (continuación)

\begin{tabular}{|c|c|c|c|c|}
\hline ÍTEM & $\begin{array}{c}\% \text { POR } \\
\text { ALTERNATIVA }\end{array}$ & $\begin{array}{l}\% \text { POR ALTERNATIVA } \\
\text { H** }^{*}\end{array}$ & $\begin{array}{l}\text { \% POR ALTERNATIVA } \\
M^{* * *}\end{array}$ & $\mathbf{P}^{*}$ \\
\hline Nerviosismo (48) & & & & 0,3434 \\
\hline 0. No & 28,00 & 9,33 & 18,67 & \\
\hline 1. Sí & 72,00 & 20,00 & 52,00 & \\
\hline Angustia (49) & & & & 0,6449 \\
\hline 0. No & 45,67 & 14,00 & 31,67 & \\
\hline 1. Sí & 54,33 & 15,33 & 39,00 & \\
\hline Disnea (50) & & & & 0,0375 \\
\hline 0. No & 59,00 & 20,00 & 39,00 & \\
\hline 1. Sí & 41,00 & 9,33 & 31,67 & \\
\hline Grupo (51) & & & & 0,781 \\
\hline 0. No & 59,00 & 17,67 & 41,33 & \\
\hline 1. Sí & 41,00 & 11,67 & 29,33 & \\
\hline Calle (52) & & & & 0,003 \\
\hline 0. No & 67,00 & 23,33 & 43,67 & \\
\hline 1. Sí & 33,00 & 6,00 & 27,00 & \\
\hline Experiencia positiva - Orgullo (57) & & & & 0,8846 \\
\hline 0. No & 29,00 & 8,33 & 20,67 & \\
\hline 1. Sí & 71,00 & 21,00 & 50,00 & \\
\hline Experiencia negativa - Abrumado (58) & & & & 0,1675 \\
\hline 0. Sí & 65,00 & 17,33 & 47,67 & \\
\hline 1. No & 35,00 & 12.00 & 23,00 & \\
\hline Experiencia positiva - Aprender (59) & & & & 0,7721 \\
\hline 0. No & 35,33 & 10,00 & 25,33 & \\
\hline 1. Sí & 64,67 & 19,33 & 45,33 & \\
\hline $\begin{array}{l}\text { Experiencia negativa - Menospreciado } \\
\text { (60) }\end{array}$ & & & & 0,6456 \\
\hline 0. Sí & 25,67 & 7,00 & 18,67 & \\
\hline 1. No & 74,33 & 22,33 & 52,00 & \\
\hline
\end{tabular}

*Prueba U de Mann-Whitney

**Hombres

****Mujeres

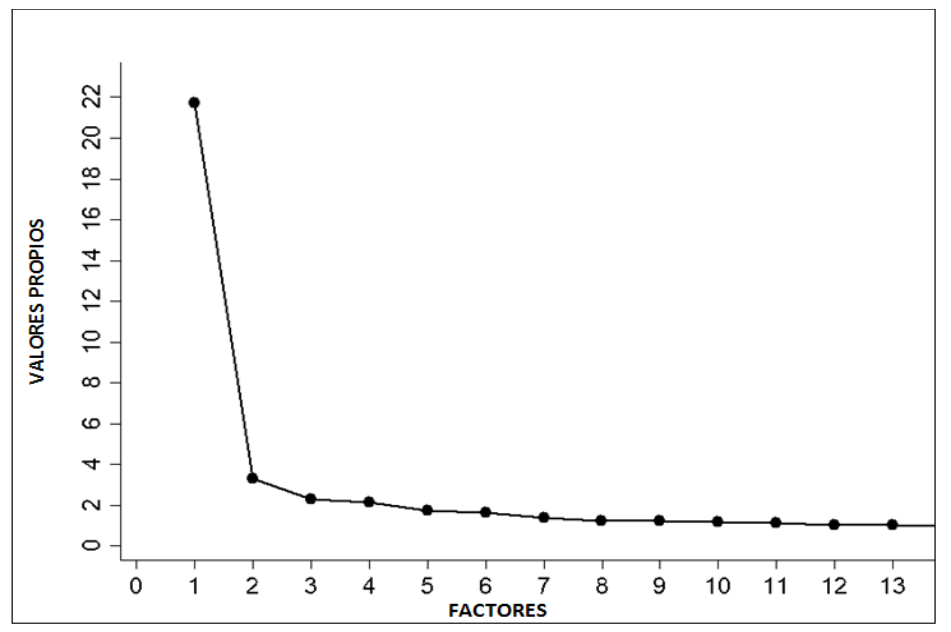

Gráfico 1. Gráfico de sedimentación de Cattel 
Tabla 6. Carga factorial policórica y correlación policórica con CGI-S.

\begin{tabular}{|c|c|c|c|}
\hline Ítem & Descripción & $\begin{array}{l}\text { Carga factorial } \\
\text { (Factor "salud") }\end{array}$ & $\begin{array}{l}\text { Correlación } \\
\text { con CGI-S }\end{array}$ \\
\hline \multicolumn{4}{|l|}{ WHODAS 2.0 } \\
\hline Estar de pie (01) & Dificultad para mantenerse de pie & 0,4867 & $-0,2674$ \\
\hline Quehaceres (02) & Dificultad para cumplir con quehaceres de la casa & 0,6212 & $-0,3968$ \\
\hline Aprender una nueva tarea $(03)$ & Dificultad para aprender una nueva tarea & 0,6405 & $-0,3923$ \\
\hline Problemas en comunidades (04) & $\begin{array}{l}\text { Dificultad para participar en actividades de la } \\
\text { comunidad }\end{array}$ & 0,4681 & $-0,2038$ \\
\hline $\begin{array}{l}\text { Haber sido afectado } \\
\text { emocionalmente }(05)\end{array}$ & Afectación emocional por condición de salud & 0,5839 & $-0,3304$ \\
\hline Concentrarse (06) & Dificultad para concentrarse & 0,6943 & $-0,3362$ \\
\hline Andar (07) & Dificultad para caminar largas distancias & 0,5181 & $-0,1371$ \\
\hline Lavarse (08) & Dificultad para bañarse & 0,5091 & $-0,2504$ \\
\hline Vestirse (09) & Dificultad para vestirse & 0,5783 & $-0,2855$ \\
\hline Relacionarse con desconocidos (10) & Dificultad para relacionarse con personas nuevas & 0,6151 & $-0,2708$ \\
\hline Mantener amistad (11) & Dificultad para mantener una amistad & 0,5569 & $-0,2602$ \\
\hline $\begin{array}{l}\text { Trabajo diario / Actividades } \\
\text { escolares (12) }\end{array}$ & Dificultad para trabajar / estudiar & 0,6073 & $-0,2640$ \\
\hline \multicolumn{4}{|l|}{ WHOQOL-BREF } \\
\hline Dependencia de medicinas (13) & Necesidad de tratamiento para funcionar & 0,5825 & $-0,2026$ \\
\hline Sentimientos positivos (14) & Disfrute de la vida & 0,6506 & $-0,3560$ \\
\hline $\begin{array}{l}\text { Espiritualidad, religión, creencias } \\
\text { personales (15) }\end{array}$ & Sentido de la vida & 0,6936 & $-0,3925$ \\
\hline $\begin{array}{l}\text { Pensamiento, aprendizaje, memoria, } \\
\text { concentración (16) }\end{array}$ & Capacidad de concentración & 0,5766 & $-0,3388$ \\
\hline Libertad y seguridad (17) & Sensación de seguridad & 0,7402 & $-0,4011$ \\
\hline Ambiente físico (18) & Ambiente físico saludable & 0,4888 & $-0,2983$ \\
\hline Imagen corporal (19) & Aceptación de apariencia física & 0,4874 & $-0,2132$ \\
\hline Recursos económicos (20) & Disponibilidad de dinero para necesidades & 0,3511 & $-0,1664$ \\
\hline Oportunidad de información (21) & Disponibilidad de información & 0,4718 & $-0,3168$ \\
\hline Ocio y descanso (22) & Oportunidad para actividades de ocio & 0,077 & 0,0042 \\
\hline Movilidad (23) & Capacidad de desplazarse & 0,4635 & $-0,3585$ \\
\hline Sueño y descanso (24) & Satisfacción con su sueño & 0,4669 & $-0,3567$ \\
\hline Relaciones personales (25) & Satisfacción con relaciones personales & 0,6442 & $-0,3844$ \\
\hline Actividad sexual (26) & Satisfacción con vida sexual & 0,4836 & $-0,3333$ \\
\hline Hogar (27) & Satisfacción con condiciones de vivienda & 0,4623 & $-0,1658$ \\
\hline Atención sanitaria / social (28) & $\begin{array}{l}\text { Satisfacción con disponibilidad de servicios de } \\
\text { salud }\end{array}$ & 0,3 & $-0,0810$ \\
\hline Transporte (29) & Satisfacción con servicios de transporte & 0,1491 & $-0,0931$ \\
\hline Sentimientos negativos (30) & Frecuencia de sentimientos negativos & 0,7595 & $-0,4269$ \\
\hline \multicolumn{4}{|l|}{ Índice de Calidad de Vida } \\
\hline Bienestar físico (31) & $\begin{array}{l}\text { Contar con energía, ausencia de dolor y problemas } \\
\text { físicos }\end{array}$ & 0,669 & $-0,3785$ \\
\hline \multirow[t]{2}{*}{ Bienestar psicológico (32) } & $\begin{array}{l}\text { Bienestar psicológico o emocional, sentirse bien } \\
\text { consigo mismo }\end{array}$ & 0,7891 & $-0,4507$ \\
\hline & & & Continúa. \\
\hline
\end{tabular}


Selección de ítems para el desarrollo de un instrumento de evaluación de severidad de trastornos mentales.

Tabla 6. Carga factorial policórica y correlación policórica con CGI-S. (continuación)

\begin{tabular}{|c|c|c|c|}
\hline Ítem & Descripción & $\begin{array}{l}\text { Carga factorial } \\
\text { (Factor "salud") }\end{array}$ & $\begin{array}{l}\text { Correlación } \\
\text { con CGI-S }\end{array}$ \\
\hline Autocuidado (33) & $\begin{array}{l}\text { Independencia, cuidar de uno mismo y tomar } \\
\text { propias decisiones }\end{array}$ & 0,6936 & $-0,3314$ \\
\hline Funcionamiento ocupacional (34) & $\begin{array}{l}\text { Capacidad de realizar trabajo, estudio o actividades } \\
\text { del hogar }\end{array}$ & 0,6751 & $-0,3168$ \\
\hline Funcionamiento interpersonal (35) & Capacidad de responder y relacionarse & 0,7214 & $-0,3780$ \\
\hline Apoyo social-emocional (36) & Contar con personas que brinden ayuda emocional & 0,5838 & $-0,3056$ \\
\hline $\begin{array}{l}\text { Apoyo comunitario y de servicios } \\
\text { (37) }\end{array}$ & Disponer de apoyo por parte de vecinos & 0,5538 & $-0,2589$ \\
\hline Plenitud personal (38) & Realización personal y cumplimiento de metas & 0,6869 & $-0,4073$ \\
\hline Satisfacción espiritual (39) & $\begin{array}{l}\text { Actitud espiritual hacia más allá de lo material, paz } \\
\text { consigo mismo y los demás }\end{array}$ & 0,6113 & $-0,3844$ \\
\hline Calidad de vida global (40) & Satisfacción y felicidad con su vida en general & 0,7958 & $-0,4275$ \\
\hline \multicolumn{4}{|c|}{ Medidas de síntomas transversales del DSM-5 } \\
\hline Enfado (41) & Sensación de enfado, o irritabilidad & 0,5645 & $-0,4409$ \\
\hline Suicidalidad (42) & Ideas de dañarse a sí mismo & 0,7147 & $-0,4766$ \\
\hline Sueño (43) & Presencia de problemas de sueño & 0,5248 & $-0,3470$ \\
\hline \multicolumn{4}{|l|}{ Depresión (EPD) } \\
\hline Cansado (44) & Sensación de cansancio & 0,7251 & $-0,3877$ \\
\hline Anhedonia (45) & Ausencia de gusto o satisfacción & 0,6065 & $-0,4051$ \\
\hline Tristeza (46) & Sensación de tristeza & 0,7601 & $-0,4613$ \\
\hline Reactividad (47) & Mejoría de ánimo frente a estímulos positivos & 0,5702 & $-0,3301$ \\
\hline \multicolumn{4}{|l|}{ Escala de Ansiedad de Lima } \\
\hline Nerviosismo (48) & Tendencia a sentirse nervioso & 0,6533 & $-0,3906$ \\
\hline Angustia (49) & Sensación de angustia, tener los nervios de punta & 0,7501 & $-0,4824$ \\
\hline Disnea (50) & Sensación de falta de aire o ahogo & 0,5984 & $-0,5414$ \\
\hline Grupo (51) & $\begin{array}{l}\text { Incomodidad para acercarse a grupos de personas } \\
\text { e interactuar }\end{array}$ & 0,6509 & $-0,3412$ \\
\hline Calle (52) & Miedo a caminar por la calle & 0,5051 & $-0,5149$ \\
\hline \multicolumn{4}{|l|}{ Pemberton Happiness Index } \\
\hline Sentido de vida (53) & Creer que su vida es útil y valiosa & 0,6998 & $-0,4161$ \\
\hline Crecimiento personal (54) & Crecer con aprendizajes y desafíos & 0,6987 & $-0,4198$ \\
\hline Control percibido (55) & Sentirse capaz de resolver problemas & 0,7558 & $-0,3793$ \\
\hline Autonomía (56) & Poder ser uno mismo & 0,7179 & $-0,3782$ \\
\hline $\begin{array}{l}\text { Experiencia positiva - } \\
\text { Orgullo (57) }\end{array}$ & Orgullo o satisfacción por lo que hizo & 0,5577 & $-0,3516$ \\
\hline $\begin{array}{l}\text { Experiencia negativa - } \\
\text { Abrumado (58) }\end{array}$ & Sentirse abrumado & 0,6007 & $-0,3457$ \\
\hline $\begin{array}{l}\text { Experiencia positiva - } \\
\text { Aprender (59) }\end{array}$ & Aprender algo interesante & 0,4235 & $-0,3192$ \\
\hline $\begin{array}{l}\text { Experiencia negativa - } \\
\text { Menospreciado }(60)\end{array}$ & Sentirse menospreciado & 0,5249 & $-0,4064$ \\
\hline
\end{tabular}


Tabla 7. Ítems seleccionados

\begin{tabular}{|c|c|c|c|c|c|c|}
\hline \multicolumn{4}{|c|}{$\begin{array}{c}17 \text { variables con mayor cCGI ordenadas en base a } \\
\text { CFs }\end{array}$} & \multicolumn{3}{|c|}{$\begin{array}{c}17 \text { variables con mayor CF ordenadas en base } \\
\text { a cCGIs }\end{array}$} \\
\hline $\mathbf{N}$ & Ítem & cCGI & $\mathbf{C F}$ & $\mathbf{C F}$ & cCGI & Ítem \\
\hline 1 & Calidad de vida global (40) & $-0,4275$ & 0,7958 & 0,7501 & $-0,4824$ & Angustia (49) \\
\hline 2 & Bienestar psicológico (32) & $-0,4507$ & 0,7891 & 0,7147 & $-0,4766$ & Suicidalidad (42) \\
\hline 3 & Tristeza (46) & $-0,4613$ & 0,7601 & 0,7601 & $-0,4613$ & Tristeza (46) \\
\hline 4 & Sentimientos negativos (30) & $-0,4269$ & 0,7595 & 0,7891 & $-0,4507$ & Bienestar psicológico (32) \\
\hline 5 & Angustia (49) & $-0,4824$ & 0,7501 & 0,7958 & $-0,4275$ & Calidad de vida global (40) \\
\hline 6 & Libertad y seguridad (17) & $-0,4011$ & 0,7402 & 0,7595 & $-0,4269$ & Sentimientos negativos (30) \\
\hline 7 & Suicidalidad (42) & $-0,4766$ & 0,7147 & 0,6987 & $-0,4198$ & Crecimiento personal (54) \\
\hline 8 & Sentido de vida (53) & $-0,4161$ & 0,6998 & 0,6998 & $-0,4161$ & Sentido de vida (53) \\
\hline 9 & $\begin{array}{l}\text { Crecimiento } \\
\text { personal (54) }\end{array}$ & $-0,4198$ & 0,6987 & 369 & $-0,4073$ & Plenitud personal (38) \\
\hline 10 & $\begin{array}{l}\text { Espiritualidad, religión, } \\
\text { creencias personales (15) }\end{array}$ & $-0,3925$ & 0,6936 & 0,7402 & $-0,4011$ & Libertad y seguridad (17) \\
\hline 11 & Plenitud personal (38) & $-0,4073$ & 0,6869 & 0,6936 & $-0,3925$ & $\begin{array}{l}\text { Espiritualidad, religión, creencic } \\
\text { personales }(15)\end{array}$ \\
\hline 12 & Quehaceres (02) & $-0,3968$ & 0,6212 & 0,7251 & $-0,3877$ & Cansado (44) \\
\hline 13 & Anhedonia (45) & $-0,40$ & 0,6 & 8 & $-0,3793$ & $\begin{array}{l}\text { Control } \\
\text { Percibido (55) }\end{array}$ \\
\hline 14 & Disnea (50) & $-0,5414$ & 0,5984 & 0,7179 & $-0,3782$ & Autonomía (56) \\
\hline 15 & Enfado (41) & $-0,4409$ & 0,5645 & 0,7214 & $-0,3780$ & $\begin{array}{l}\text { Funcionamiento interpersonal } \\
\text { (35) }\end{array}$ \\
\hline 16 & $\begin{array}{l}\text { Experiencia negativa - } \\
\text { Menospreciado (60) }\end{array}$ & $-0,4064$ & 0,5249 & 0,6943 & $-0,3362$ & Concentrarse (06) \\
\hline 17 & Calle (52) & $-0,5149$ & 0,5051 & 0,6912 & $-0,3314$ & Autocuidado (33) \\
\hline
\end{tabular}

cCGI: Correlación con CGI-S.

CF: Carga factorial (factor "salud").

\section{DISCUSIÓN}

El presente estudio ha descrito la aplicación de 60 ítems con el objetivo de encontrar los más relevantes en la evaluación de trastornos psiquiátricos basándose en los conceptos de calidad de vida, discapacidad, bienestar y severidad de sintomatología (28).

Se utilizaron 7 distintas escalas seleccionadas tomando en cuenta la amplitud y complejidad del concepto de severidad y la importancia que ha alcanzado la funcionalidad y la calidad de vida dentro de éste, buscando que los ítems seleccionados de cada uno de estos instrumentos complementará a los otros aportando a la evaluación del constructo de salud (29).

En la muestra de 313 pacientes se encontró una distribución de variables socio-demográficas similar a la hallada en los estudios de selección y validación, de las escalas utilizadas $(20,21,2325,30,31,32)$.
Con respecto a dichas variables demográficas, se encontró una relación significativa con el sexo en 1 de los 11 ítems considerados como los más relevantes, el ítem número 17 , el cual evalúa sensación de seguridad, ocurriendo que los pacientes de sexo masculino presentaron puntajes más elevados, percibiendo tener mayor seguridad.

Por medio del análisis factorial exploratorio se halló un modelo unifactorial en base a la prueba de sedimentación de Cattel (27); a este único factor hemos denominado "salud".

Utilizando las cargas factoriales y los coeficientes de correlación con el puntaje de la CGI-S asignado por el psiquiatra tratante se seleccionaron los 11 ítems más relevantes, los cuales son: Calidad de vida global, Bienestar psicológico, Tristeza, Sentimientos negativos, Angustia, Libertad y seguridad, Suicidalidad, Sentido de vida, Crecimiento personal, Espiritualidad, religión y creencias personales y 
Selección de ítems para el desarrollo de un instrumento de evaluación de severidad de trastornos mentales.

\section{Plenitud personal.}

Del ICV cabe resaltar 3 ítems con altos valores tanto en la correlación con la CGI-S como en CF, por lo que fueron considerados relevantes: Calidad de vida global, Bienestar psicológico y Plenitud personal. La Calidad de vida global tuvo la CF más alta de los ítems evaluados con 0,7958 y una correlación de $-0,4275$ con la CGI-S; Bienestar psicológico tuvo una CF de 0,7891, siendo el segundo ítem con CF más alta, además de tener un coeficiente de correlación de $-0,4507$ con la impresión clínica del psiquiatra; y la plenitud personal, un coeficiente de correlación de $-0,4073$ y CF de 0,6869 . En concordancia con esto, en la presentación y validación original del ICV estos tres ítems tuvieron las 3 mayores CFs, teniendo la Calidad de vida global la más alta $(\mathrm{CF}=0,86)$, seguido por Bienestar psicológico $(\mathrm{CF}=0,84)$ y Plenitud personal $(\mathrm{CF}=0,81)(20)$.

Tomando en cuenta el poder psicométrico del ítem Calidad de vida global podría plantearse su uso como pregunta única para la autoevaluación de la severidad de la enfermedad mental, sin embargo al validarse el ICV en una muestra peruana, se encontró que tanto al proponer un modelo unifactorial como uno de dos componentes, dicho ítem no fue el más relevante, siendo superado en $\mathrm{CF}$ por Bienestar psicológico y Plenitud personal, asumiendo un solo factor; y al asumir dos factores, fue el ítem con menor CF en la evaluación del primer dominio y fue superado por Satisfacción espiritual en la evaluación del segundo factor (33). Por su potencial para funcionar como un indicador general de severidad y por las razones dadas, se considera que sería necesario realizar mayores estudios para evaluar sus propiedades especialmente como ítem único.

En el WHOQOL-BREF se encontraron tres ítems relevantes, los cuales evaluaban la presencia de Sentimientos negativos; Libertad y seguridad; y finalmente, la Espiritualidad, religión y creencias personales. Estos tuvieron CFs de 0,7595, 0,7402 y 0,6936; y además, presentaron correlaciones de $-0,4269,-0,4011$ y $-0,3925$ con la impresión clínica del médico, respectivamente.

Durante las pruebas de campo del WHOQOLBREF se plantearon cuatro dominios: calidad de vida física, psicológica, social y ambiental; la evaluación de seguridad demostró ser uno de los dos mejores ítems en la evaluación del dominio de calidad de vida ambiental, con una correlación de 0,64 con dicho dominio. La espiritualidad tuvo una correlación de 0,70 con el factor de bienestar psicológico en las pruebas de campo del WHOQOL, siendo uno de los cuatro mejores ítems en la evaluación de dicho factor, el cual además demostró ser el más relevante de los cuatro dominios (34). La evaluación de los Sentimientos negativos no presentó correlaciones muy elevadas con ninguno de los cuatro dominios, sin embargo este ítem demostró las más altas correlaciones con los puntajes del Symptom Checklist-90 para ansiedad, ansiedad fóbica y depresión, en la validación del WHOQOL no abreviado en una población holandesa (35).

En el PHI se encuentra compuesto por ítems que evalúan el bienestar experimentado, dividido en eventos positivos y negativos, e ítems que evalúan el bienestar recordado, compuesto por cuatro dominios: bienestar general, bienestar hedónico, bienestar eudaimónico y bienestar social. En este instrumento se encontraron 4 ítems relevantes, de los cuales 2 estuvieron entre los 11 ítems seleccionados. Significado de vida y Crecimiento personal tuvieron CF de 0,6998 y 0,6987 con coeficientes de correlación con la CGI-S de $-0,4161$ y $-0,4198$, respectivamente. Significado de vida, en la elaboración y validación del PHI, presentó una $\mathrm{CF}$ de 0,60 en el dominio de bienestar eudaimónico, siendo éste el segundo valor más alto, además de ser el ítem que al ser retirado ocasionó la segunda mayor disminución de consistencia interna. Crecimiento personal fue el cuarto ítem con mayor $\mathrm{CF}$ en el dominio de bienestar eudaimónico, con 0,50 (21).

En la EPD se seleccionó un ítem, el cual hace referencia a la tristeza, teniendo una $\mathrm{CF}$ de 0,7601 y un coeficiente de correlación con la CGI de -0,4623. La EPD tiene versiones cortas de 4 (EPD-4) y 6 ítems (EPD-6); la EPD-4 tiene estructura unifactorial, mientras que en la EPD-6 se identifican 2 factores: "afecto y sueño" y "corporal". La Tristeza en la EPD6 fue el ítem que más aportó a la dimensión sobre "afecto y sueño"; y en la EPD-4, tuvo la segunda CF más alta con respecto al único factor (24).

De los 5 ítems de EAL utilizados en este estudio, se seleccionó el ítem sobre Angustia, el cual tuvo una CF de 0,7501 y una correlación con la impresión clínica de $-0,4824$. En el estudio que describe la construcción de la versión de 20 ítems de la EAL, la Angustia fue el ítem más relevante asumiendo un modelo unifactorial, con una CF de 0,81 , siendo este valor el más alto (23). 
Por otra parte, resultó seleccionado el ítem de las medidas transversales del DSM-5 que evaluaba Suicidalidad, el cual tuvo una CF de 0,7147 y un coeficiente de correlación con la CGI-S de -0,4766; dicho ítem durante las pruebas de campo de las medidas transversales auto-reportadas del DSM-5 en adultos tuvo una confiabilidad test-retest, basada en coeficientes de correlación intraclase agrupados, de 0,77 ; siendo éste el quinto valor más alto entre los 23 ítems postulados (22).

Con respecto al WHODAS 2.0 de 12 ítems, en el presente estudio el ítem sobre Dificultad para concentrarse estuvo entre los 17 ítems con mayor CF. Dicho ítem ha presentado en estudios previos una $\mathrm{CF}$ de 0,54 en el dominio de "entendimiento y comunicación" en pacientes con enfermedades crónicas (17) y una CF de 0,683 en pacientes con trastornos del espectro ansioso-depresivo, cargas que lo colocan como el quinto mejor ítem de 12 en evaluar dicho dominio. Sin embargo, por demostrar una baja correlación con la CGI, -0,3362, no fue incluido entre los 11 ítems más relevantes (30).

Podemos inferir que los ítems seleccionados han presentado una mayor carga debido a que la mayoría evalúa indirectamente la presencia de síntomas de las patologías más predominantes, además de valorar la satisfacción del paciente con su vida, lo que espera de ella, y el efecto de su enfermedad en ésta.

En cuanto a las limitaciones de nuestro estudio, debemos considerar que los resultados han sido obtenidos a partir de una muestra de pacientes procedentes, en términos generales, de un nivel socio-económico, entorno cultural y sistema de valores similares, por lo que el concepto de severidad propuesto ha sido formado en base a éstos y podría variar al ser aplicados los ítems a una población diferente, como por ejemplo, los habitantes de una zona rural o urbano-marginal.

Los ítems Sentido de vida y Espiritualidad, religión y creencias personales tuvieron un buen desempeño psicométrico, hallándose entre los 11 ítems seleccionados, mientras que ítems como Funcionamiento ocupacional y Recursos económicos no alcanzaron el punto de corte, hallazgo que podría ser llamativo considerando la condición socio-económica de la mayoría de la población peruana; para lo se plantean tres posibles explicaciones. La primera es que al tratarse de una muestra compuesta exclusivamente de individuos con trastornos psiquiátricos, el sentido de vida cobre mayor importancia, al tener estos pacientes un sistema de valores diferente al de la población general. La segunda es que ambos reactivos (sentido de vida y espiritualidad, religión y creencias personales) midan de forma indirecta carencias o necesidades insatisfechas; como se observó en el caso del ítem Satisfacción espiritual que tuvo puntajes significativamente mayores en personas con menor pobreza en la validación del ICV en una muestra peruana (19). La tercera es que al no haber tomado como dato demográfico la presencia de necesidades básicas insatisfechas se haya pasado por alto la predominancia de cierto grupo socio-económico en la muestra, ocasionando que ciertos ítems pierdan potencia.

Asimismo, la predominancia de diagnósticos de trastornos del espectro ansioso-depresivo (82,87\%) podría ser una limitación, porque se podría conjeturar que haya aumentado la potencia de ciertos reactivos dirigidos a la evaluación de dichas patologías.

Otra limitación a tomar en cuenta es la subjetividad detrás la CGI-S que se utilizó como gold standard. Este instrumento, al basarse en la opinión del médico tratante está sujeto a su experiencia y no cuenta con una estandarización de lo que cada distinto psiquiatra evaluador podría considerar como severo o disfuncional.

Finalmente los resultados no necesariamente son aplicables a pacientes con psicosis o déficit cognitivo (los cuales fueron excluidos); y asimismo, los ítems seleccionados deben ser evaluados psicométricamente en futuros estudios para verificar su utilidad como escala para la medición global de la salud mental.

En conclusión, en base a los resultados del análisis factorial y la correlación con la severidad de la sintomatología evaluada por el psiquiatra del paciente, 11 ítems fueron seleccionados: Calidad de vida global, Bienestar psicológico, Tristeza, Sentimientos negativos, Angustia, Libertad y seguridad, Suicidalidad, Sentido de vida, Crecimiento personal, Espiritualidad, religión y creencias personales y Plenitud personal. Estos ítems podrían constituir un instrumento corto, de auto-llenado para evaluación del posible constructo de salud.

\section{Declaración de financiamiento y conflicto de interés:} El presente estudio fue financiado por los autores. Los autores afirman no tener conflictos de interés. 
Selección de ítems para el desarrollo de un instrumento de evaluación de severidad de trastornos mentales.

\section{Correspondencia:}

Percy Gustavo Damiani Rodríguez

José Sabogal 220. Miraflores, Lima, Perú.

Correo electrónico: percy.damiani.r@upch.pe

\section{REFERENCIAS BIBLIOGRÁFICAS}

1. Valdez W, Miranda JA. Carga de enfermedad en el Perú, estimación de los años de vida saludables perdidos 2012. Lima: Dirección general de epidemiología; 2014. p. 29-31.

2. Piazza M, Fiestas F. Prevalencia anual de trastornos y uso de servicios de salud mental en el Perú: resultados del estudio mundial de salud mental, 2005. Rev Peru Med Exp Salud Publica. 2014; 31(1):30-38. DOI: http://dx.doi.org/10.17843/rpmesp.2014.311.5

3. Instituto Nacional de Salud Mental. Estudio Epidemiológico de Salud Mental en Lima Metropolitana y Callao - replicación 2012. Informe general. Anales de Salud Mental. 2013; 29(1):23-192.

4. Busner J, Targum SD. The Clinical Global Impressions Scale: applying a research tool in clinical practice. Psychiatry. 2007; 4(7):28-37.

5. Charlwood P, Mason A, Goldacre M, Cleary R, Wilkinson E (eds). Health Outcome Indicators: Severe Mental Illness. Oxford: Report of a working group to the Department of Health; 1999.

6. Kessler RC, Chiu WT, Demler O, Merikangas KR, Walters EE. Prevalence, severity, and comorbidity of 12-month DSM-IV disorders in the National Comorbidity Survey Replication. Arch Gen Psychiatry. 2005;62(6):617-27.

7. Ruggeri $M$, Leese $M$, Thornicroft $G$, Bisoffi $G$, Tansella M. Definition and prevalence of severe and persistent mental illness. $\mathrm{Br} \quad \mathrm{J}$ Psychiatry. 2000;177:149-55.

8. Demyttenaere K, Bruffaerts R, Posada-Villa J, Gasquet I, Kovess V, Lepine JP, et al. Prevalence, severity, and unmet need for treatment of mental disorders in the World Health Organization World Mental Health Surveys. JAMA. 2004;291(21):258190.

9. Center for Behavioral Health Statistics and Quality. 2016 National Survey on Drug Use and Health: Methodological summary and definitions. Rockville, MD: Substance Abuse and Mental Health Services Administration; 2017.

10. World Health Organization. Helping people with severe mental disorders live longer and healthier lives: policy brief. Ginebra: World Health Organization; 2017. (Fecha de acceso 24 de marzo del 2019) Disponible en: http://www.who.int/iris/ handle $/ 10665 / 259575$

11. National Institute of Mental Health. Mental Illness. Washington DC: National Institute of Mental Health;
2017. (Fecha de acceso 24 de julio del 2018)

Disponible en: https://www.nimh.nih.gov/health/ statistics/mental-illness.shtml

12. Cucciare M, O’Donohue W, Cummings N. Practical Resources for the Mental Health Professional: Evidence-Based Adjunctive Treatments. Elsevier Academic Press; 2008.

13. Clarke DE, Kuhl EA. DSM-5 cross-cutting symptom measures: a step towards the future of psychiatric care? World Psychiatry. 2014; 13(3):314-6. doi: 10.1002/wps. 20154

14. World Health Organization. How to use the ICF: A practical manual for using the International Classification of Functioning, Disability and Health (ICF). Geneva: World Health Organization; 2013.

15. Development of the World Health Organization WHOQOL-BREF quality of life assessment. The WHOQOL Group. Psychol Med. 1998; 28(3):551-8.

16. Stambor Z. Changing health care's focus. Monitor on Psychologi. 2006; 37(1):42.

17. Garin O, Ayuso-Mateos JL, Almansa J, Nieto M, Chatterji S, Vilagut G, et al. Validation of the "World Health Organization Disability Assessment Schedule, WHODAS-2" in patients with chronic diseases. Health Qual Life Outcomes. 2010; 8:5. doi: 10.1186/14777525-8-51

18. Najafi M, Sheikhvatan M, Montazeri A, Sheikhfatollahi M. Factor structure of the World Health Organization's Quality of Life QuestionnaireBREF in patients with coronary artery disease. Int $\mathrm{J}$ Prev Med. 2013;4(9):1052-8.

19. Saavedra JE, Mezzich JE, Sanez Y, Padilla M, Mejía O. Índice de Calidad de Vida: Validación en una muestra peruana. Anales de Salud Mental. 2010; 26(2):33-43.

20. Mezzich JE, Cohen NL, Ruiperez MA, Banzato CEM, Zapata-Vega MI. The Multicultural Quality of Life Index: presentation and validation. J Eval Clin Pract. 2011;17(2):357-64.

21. Hervás G, Vázquez C. Construction and validation of a measure of integrative well-being in seven languages: the Pemberton Happiness Index. Health Qual Life Outcomes. 2013;11:66. doi: 10.1186/14777525-11-66

22. Narrow WE, Clarke DE, Kuramoto SJ, Kraemer HC, Kupfer DJ, Greiner L, et al. DSM-5 field trials in the United States and Canada, Part III: development and reliability testing of a cross-cutting symptom assessment for DSM-5. Am J Psychiatry. 2013;170(1):71-82. DOI: 10.1176/appi.ajp.2012. 12071000

23. Lozano-Vargas A, Vega-Dienstmaier JM. Construcción y propiedades psicométricas de la Escala de Ansiedad de Lima de 20 ítems (EAL-20). Rev Neuropsiquiatr. 2019;81(4):226-234. DOI: http://dx.doi.org/10.20453/rnp.v81i4.3437 
24. Vega-Dienstmaier JM. Construcción de versiones cortas de la Escala de Psicopatología Depresiva para tamizaje de depresión mayor y sus características psicométricas. Rev Neuropsiquiatr. 2018;81(3):157161. DOI: https://doi.org/10.20453/rnp.v81i3.3383

25. Vega Dienstmaier JM. Estructura de los síndromes afectivos y ansiosos en pacientes psiquiátricos ambulatorios de un hospital general. Tesis de Maestría. Lima, Perú: Universidad Peruana Cayetano Heredia; 2017. (Fecha de acceso 24 de febrero del 2019) Disponible en: http://repositorio.upch.edu.pe/ handle/upch/1032

26. Comrey AL, Lee HB. A first course in factor analysis. Abingdon: Psychology Press; 2013.

27. Cattell RB. The scree test for the number of factors. Multivariate Behav Res. 1966;1(2):245-76. DOI: $10.1207 / \mathrm{s} 15327906 \mathrm{mbr} 0102 \_10$

28. Griffiths C. Determinates of mental illness severity. International Journal of Psychosocial Rehabilitation. 2017; 21 (2): 31-34. (Fecha de acceso 24 de febrero del 2019) Disponible en: http://www.psychosocial. com/IJPR_21/Determinants_Griffiths.html

29. Gonzalez AI, Seijo N, Díaz JC, Lopez E, Santed MA. Revisiting the concept of severe mental illness: severity indicators and healthcare spending in psychotic, depressive and dissociative disorders. J Ment Health. 2017;10:1-7. doi: 10.1080/09638237.2017

30. Axelsson E, Lindsäter E, Ljótsson B, Andersson E, Hedman-Lagerlöf E. The 12-item Self-Report World Health Organization Disability Assessment Schedule (WHODAS) 2.0 Administered via the internet to individuals with anxiety and stress disorders: A psychometric investigation based on data from two clinical trials. JMIR Ment Health. 2017;4(4):e58. doi: 10.2196/mental.7497
31. Krägeloh CU, Kersten P, Rex Billington D, Hsu PH-C, Shepherd D, Landon J, et al. Validation of the WHOQOL-BREF quality of life questionnaire for general use in New Zealand: confirmatory factor analysis and Rasch analysis. Qual Life Res. 2013;22(6):1451-7

32. Clarke DE, Narrow WE, Regier DA, Kuramoto SJ, Kupfer DJ, Kuhl EA, et al. DSM-5 field trials in the United States and Canada, Part I: study design, sampling strategy, implementation, and analytic approaches. Am J Psychiatry. 2013;170(1):43-58.

33. Schwartz KI, Zapata-Vega MI, Mezzich JE, Mazzotti G. Validation study of the Multicultural Quality of Life Index (MQLI) in a Peruvian sample. Braz J Psychiatry. 2006; 28(1):24-8.

34. Skevington SM, Lotfy M, O'Connell KA, WHOQOL Group. The World Health Organization's WHOQOLBREF quality of life assessment: psychometric properties and results of the international field trial. A report from the WHOQOL group. Qual Life Res. 2004; 13(2): 299-310.

35. Masthoff ED, Trompenaars FJ, Van Heck GL, Hodiamont PP, De Vries J. Validation of the WHO Quality of Life assessment instrument (WHOQOL-100) in a population of Dutch adult psychiatric outpatients. Eur Psychiatry. 2005;20(7):465-73.
Recibido: 25/03/2019

Aceptado: 31/03/2019 